\title{
Boosting Dissolution-Dynamic Nuclear Polarization by Multiple-Step Dipolar Order Mediated ${ }^{1} \mathrm{H} \rightarrow{ }^{13} \mathrm{C}$ Cross-Polarization
}

\author{
Stuart J. Elliott ${ }^{\mathrm{a}, \mathrm{b}, \dagger}$, Olivier Cala ${ }^{\mathrm{a}}$, Quentin Chappuis ${ }^{\mathrm{a}}$, Samuel F. Cousin ${ }^{\mathrm{a}, \mathrm{c}}$, Morgan Ceillier ${ }^{\mathrm{a}}$, Venita Decker $^{\mathrm{d}}$ and Sami Jannin $^{\mathrm{a}}$ \\ ${ }^{a}$ Centre de Résonance Magnétique Nucléaire à Très Hauts Champs - FRE 2034 Université de Lyon / CNRS / Université Claude Bernard Lyon 1 / ENS de Lyon, \\ 5 Rue de la Doua, 69100 Villeurbanne, France \\ ${ }^{\mathrm{b}}$ Department of Chemistry, Crown Street, University of Liverpool, Liverpool L69 7ZD, United Kingdom \\ Institut de Chimie Radicalaire - UMR 7273, Saint-Jérôme Campus, Av. Esc. Normandie Niemen, Aix-Marseille Université / CNRS, 13397 Marseille, Cedex 20, \\ France \\ ${ }^{\mathrm{d}}$ Bruker Biospin GMBH, Silberstriefen 4, 76289 Rheinstetten, Germany \\ † stuart-james.elliott@univ-lyon1.fr
}

\begin{abstract}
Dissolution-dynamic nuclear polarization can be boosted by employing multiplecontact cross-polarization techniques to transfer polarization from ${ }^{1} \mathrm{H}$ to ${ }^{13} \mathrm{C}$ spins. The method is efficient and significantly reduces polarization build-up times, however, it involves high-power radiofrequency pulses in a superfluid helium environment which limit its implementation and applicability and prevent a significant scaling-up of the sample size. We propose to overcome this limitation by a stepwise transfer of polarization using a low-

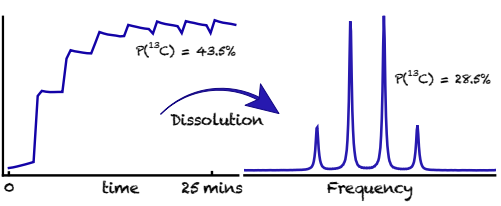
energy and low-peak power radiofrequency pulse sequence where the ${ }^{1} \mathrm{H} \rightarrow{ }^{13} \mathrm{C}$ polarization transfer is mediated by a dipolar spin order reservoir. An experimental demonstration is presented for $\left[1-{ }^{13} \mathrm{C}\right]$ sodium acetate. A solid-state ${ }^{13} \mathrm{C}$ polarization of $\sim 43.5 \%$ was achieved using this method with a build-up time constant of $\sim 5.1$ minutes, leading to a $\sim 28.5 \%{ }^{13} \mathrm{C}$ polarization in the liquidstate after sample dissolution. The low-power multiple-step polarization transfer efficiency with respect to the most advanced and highest-power multiple-contact cross-polarization approach was found to be $\sim 0.69$.
\end{abstract}

Keywords: NMR, Hyperpolarization, DNP, $d \mathrm{DNP}, \mathrm{CP}, d \mathrm{CP}$, dipolar order, dissolution

Ordinary magnetic resonance spectroscopy (MRS) and imaging (MRI) methods are often limited by the weak magnetic response from clusters of nuclear spins, even when placed within today's highest field superconducting magnets. The inherent insensitivity of a nuclear spin ensemble is engendered by the small differences in energy between nuclear spin states compared with the energy typically available at room temperature, which results in a rather flat Boltzmann distribution of nuclear spin populations.

To alleviate this issue, dissolution-dynamic nuclear polarization ( $d \mathrm{DNP}$ ) experiments are becoming increasingly employed. The hyperpolarization technique generates strong nuclear magnetic resonance (NMR) signals enhanced by factors approaching $10^{4}$ [1] for a range of nuclear spins in various media, with applications in clinical research [2-4] and metabolomics studies [5], among others [6,7]. For dilute low- $\gamma$ nuclear spins at low temperatures, the $d \mathrm{DNP}$ process [8] suffers from excessively long polarization build-up time constants $\tau_{\mathrm{DNP}}$ sometimes exceeding an hour [9].

$d$ DNP methods can be efficiently accelerated (by a factor of up to 40 ) by the implementation of radiofrequency ( $r f$ ) pulse sequences such as cross-polarization (CP) [10-17], which indirectly transfer electron spin polarization to insensitive nuclear spins ( such as ${ }^{13} \mathrm{C}$ ) via sensitive nuclear spins (such as $\left.{ }^{1} \mathrm{H}\right)$. The acceleration of the DNP process, compared with direct polarization, is attributed to the generally quicker polarization build-up timescales of proton spins at low temperatures when polarized with nitroxide radicals [18]. Multiple applications of intense $B_{1}$-matched (typ. $>15 \mathrm{kHz}$ ) simultaneous ${ }^{1} \mathrm{H}$ and ${ }^{13} \mathrm{C}$ spin-locking $r f$-fields throughout an optimized contact period (typ. $>1 \mathrm{~ms}$ ) allow the repeated indirect transfer of electron spin polarization, which is accumulated by the insensitive nuclear spins. This multiple-contact CP-DNP approach was implemented in the preparation and transportation of highly polarized metabolites [19].

Such a CP approach under $d \mathrm{DNP}$ conditions, i.e. at liquid helium temperatures, is significantly challenging, e.g. necessary high-energy and high-peak power $r f$-pulses can lead to detrimental arcing in the superfluid cryogenic bath [20]. As a result, $\mathrm{CP}$ is not widely implemented under $d \mathrm{DNP}$ conditions (typ. $T=1.0-1.6 \mathrm{~K}$ ). Such difficulties not only restrict a broader implementation of $\mathrm{CP}$, but also prevent the scaling-up of $d \mathrm{DNP}$ sample volumes required for human applications or for the parallelization of hyperpolarization [21].

We have recently demonstrated the use in a DNP context of an alternative $r f$-pulse sequence to $\mathrm{CP}$ which is of low-power, does not require synchronized $B_{1}$-matched spin-locking $r f$-fields and can ultimately overcome all previous limitations [22]. In such cases, the transfer of spin polarization is mediated by an intermediary reservoir of nuclear dipolar spin order [23-33], and even though the exact mechanism underlying the polarization transfer is yet to be fully understood, the $r f$-pulse sequence has consequently been termed dipolar order mediated crosspolarization $(d \mathrm{CP})$. It is expected that significant levels of ${ }^{13} \mathrm{C}$ polarization can be accrued if an approach incorporating consecutive $d \mathrm{CP}$ transfers can be successfully implemented, and that a significant fraction of the resulting solid-state ${ }^{13} \mathrm{C}$ polarization will be preserved upon hyperpolarization by dissolution to the liquid-state.

In the current Paper, we present an $r f$-pulse sequence containing multiple low-power polarization transfer elements in a way that is fully compatible with $d \mathrm{DNP}$ conditions. The $r f$ pulse sequence yielded a ${ }^{13} \mathrm{C}$ polarization of $\sim 43.5 \%$ with a build-up time constant $\tau_{d \mathrm{CP}}=5.1 \pm 0.2$ minutes for a sample of $\left[1-{ }^{13} \mathrm{C}\right]$ sodium acetate in the frozen solid-state, which was found to be $\sim 0.69$ of the efficiency realized by using a most advanced state-of-the-art and fully optimized multiple-contact 
$\mathrm{CP}$ experiment. We additionally investigated the dissolution of $\left[1-{ }^{13} \mathrm{C}\right]$ sodium acetate indirectly polarized via this technique, and achieved a liquid-state ${ }^{13} \mathrm{C}$ polarization level of $\sim 28.5 \%$.

Sample I (see experimental methods) was polarized by applying microwave irradiation at $f_{\mu \mathrm{w}}=198.128 \mathrm{GHz}$ (negative lobe of the EPR line) with triangular frequency modulation of amplitude $\Delta f_{\mu \mathrm{w}}= \pm 160 \mathrm{MHz}$ [34] and rate $f_{\bmod }=0.5 \mathrm{kHz}$ at a power of ca. $P_{\mu \mathrm{w}}=125 \mathrm{~mW}$ at the output of the microwave source and ca. $P_{\mu \mathrm{w}}=30 \mathrm{~mW}$ reaching the DNP cavity. Microwave gating was employed shortly before and during $d$ DNP transfer elements to allow the electron spin ensemble to return to a highly polarized state, which happens on the timescale of the longitudinal electron relaxation time (typ. $T_{1 \mathrm{e}}=$ $100 \mathrm{~ms}$ with $P_{\mathrm{e}}=99.93 \%$ under our conditions) [35]. Microwave gating provides a way to strongly attenuate paramagnetic relaxation, therefore resulting in a significant increase in the nuclear spin relaxation times in the $r f$-field (or rotating) frame. This allows the application of longer $d \mathrm{CP} r f$ pulses, which significantly increases the efficiency of nuclear spin polarization transfer.

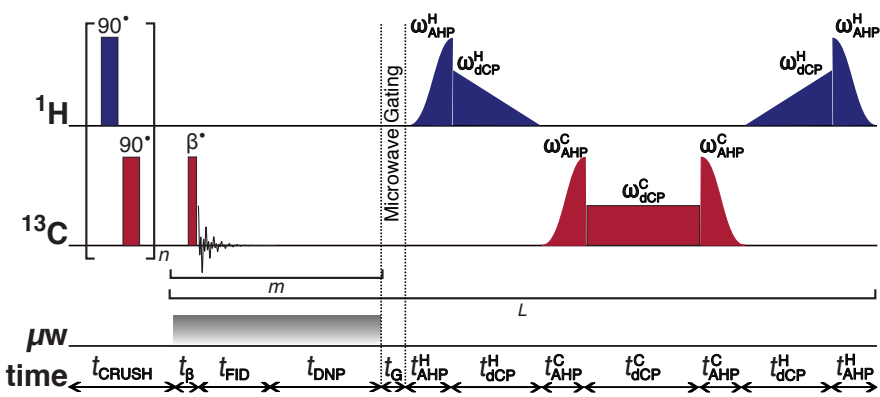

Figure 1: Schematic representation of the multiple-step dipolar order mediated cross-polarization (multi-dCP) $r f$-pulse sequence used for transferring ${ }^{1} \mathrm{H}$ Zeeman polarization to insensitive ${ }^{13} \mathrm{C}$ nuclear spins in successive steps. The experiments used the following parameters, chosen to maximize ${ }^{1} \mathrm{H} \rightarrow{ }^{13} \mathrm{C}$ polarization transfer: $n=50 ; \beta=5^{\circ} ; \tau_{\mathrm{DNP}}=30 \mathrm{~s} ; m=7 ; \tau_{\mathrm{G}}=0.5 \mathrm{~s} ; \omega_{\mathrm{AHP}}^{\mathrm{H}} / 2 \pi$ $=27.8 \mathrm{kHz} ; t_{\mathrm{AHP}}^{\mathrm{H}}=175 \mu \mathrm{s} ; \omega_{d \mathrm{CP}}^{\mathrm{H}} / 2 \pi=16.9 \mathrm{kHz} ; t_{d \mathrm{CP}}^{\mathrm{H}}=450 \mu \mathrm{s} ; \omega_{\mathrm{AHP}}^{\mathrm{C}} / 2 \pi=$ $25.8 \mathrm{kHz} ; t_{\mathrm{AHP}}^{\mathrm{C}}=175 \mu \mathrm{s} ; \omega_{d \mathrm{CP}}^{\mathrm{C}} / 2 \pi=14.6 \mathrm{kHz} ; t_{d \mathrm{CP}}^{\mathrm{C}}=49 \mathrm{~ms} ; L=8$. AHP $=$ Adiabatic Half-Passage. AHP sweep width $=100 \mathrm{kHz}$. All AHP and $d \mathrm{CP} r f$ pulses have phase $x$. The $\pi / 2$ crusher $r f$-pulses use a thirteen-step phase cycle to remove residual magnetization at the beginning of the experiment: $\{0, \pi / 18$, $5 \pi / 18, \pi / 2,4 \pi / 9,5 \pi / 18,8 \pi / 9, \pi, 10 \pi / 9,13 \pi / 9, \pi / 18,5 \pi / 3,35 \pi / 18\}$. The resonance offset was placed at the centre of the ${ }^{1} \mathrm{H}$ and ${ }^{13} \mathrm{C}$ NMR peaks.

To obtain the highest possible levels of ${ }^{13} \mathrm{C}$ polarization, it is of interest to perform multiple $d \mathrm{CP} r f$-pulse sequence cycles to incrementally transfer ${ }^{1} \mathrm{H}$ polarization to dilute ${ }^{13} \mathrm{C}$ nuclear spins embedded within the sample. Figure 1 shows such a suitable $r f$-pulse sequence capable of implementing numerous $d \mathrm{CP} \quad r f$-pulse sequence steps. Consequently, the $r f$-pulse sequence has been termed multiple-step dipolar order mediated cross-polarization (multi-dCP).

The multi-dCP $r f$-pulse sequence operates as follows:

(i) A crusher sequence of $90^{\circ} r f$-pulses with alternating phases separated by a short delay (typ. $11 \mathrm{~ms}$ ) repeated $n$ times (typ. $n=50$ ) kills residual magnetization on both $r f$-channels;

(ii) The microwave source becomes active;

(iii) The ${ }^{13} \mathrm{C}$ Zeeman magnetization trajectory is minimally perturbed by the application of a small flip-angle $r f$-pulse (typ. $\beta=5^{\circ}$ ) with a short acquisition period (typ. $t_{\mathrm{FID}}=1 \mathrm{~ms}$ ) used for detection;

(iv) ${ }^{1} \mathrm{H}$ DNP builds-up during a time $t_{\mathrm{DNP}}\left(\right.$ typ. $\left.t_{\mathrm{DNP}}=30 \mathrm{~s}\right)$; (v) Stages iii-iv are cycled $m$ times (typ. $m=7$ ) in order to monitor the evolution of the ${ }^{13} \mathrm{C}$ polarization between $d \mathrm{CP}$ steps;

(vi) The microwave source is gated and a delay of duration $t_{\mathrm{G}}=0.5 \mathrm{~s}$ occurs before the next $d \mathrm{CP}$ step, thus permitting the electron spins to relax to their highly polarized thermal equilibrium state [35];

(vii) $\mathrm{A}{ }^{1} \mathrm{H}$ adiabatic half-passage (AHP) $r f$-pulse followed by a linearly decreasing amplitude ramp ${ }^{1} \mathrm{H} r f$-pulse of amplitude $\omega_{\mathrm{dCP}}^{\mathrm{H}}$ and length $t_{\mathrm{dCP}}^{\mathrm{H}}$ presumably converts ${ }^{1} \mathrm{H}$ Zeeman polarization into ${ }^{1} \mathrm{H}-{ }^{1} \mathrm{H}$ dipolar order [36];

(viii) $\mathrm{A}{ }^{13} \mathrm{C}$ spin-locking $r f$-pulse of amplitude $\omega_{\mathrm{dCP}}^{\mathrm{C}}$ and length $t_{\mathrm{dCP}}^{\mathrm{C}}$ sandwiched between two ${ }^{13} \mathrm{C}$ AHP $r f$-pulses of opposite chronology generates ${ }^{13} \mathrm{C}$ transverse magnetization;

(ix) Stages ii-viii are repeated in $L$ units (typ. $L=8$ ) to periodically transfer ${ }^{1} \mathrm{H}$ Zeeman polarization to ${ }^{13} \mathrm{C}$ spins.

The DNP build-up time constant of ${ }^{1} \mathrm{H}$ polarization for sample I at $1.2 \mathrm{~K}$ was measured to be: $\tau_{\mathrm{DNP}}=225 \pm 1 \mathrm{~s}$ (for the positive lobe of the DNP microwave spectrum). Consequently, the period between $d \mathrm{CP}$ and $\mathrm{CP}$ polarization transfer steps was chosen to be: $t_{\mathrm{DNP}}=210 \mathrm{~s}$, corresponding to $m=7$, see Figure 1 .

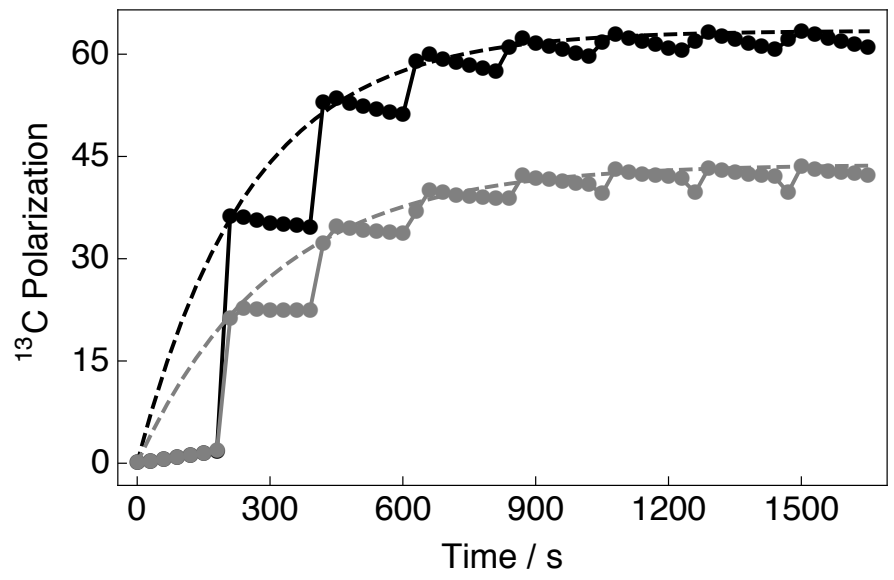

Figure 2: Experimental ${ }^{13} \mathrm{C}$ polarization build-up curves for a sample of $\mathbf{I}$ acquired at $7.05 \mathrm{~T}\left({ }^{1} \mathrm{H}\right.$ nuclear Larmor frequency $=300.13 \mathrm{MHz},{ }^{13} \mathrm{C}$ nuclear Larmor frequency $=75.47 \mathrm{MHz}$ ) and $1.2 \mathrm{~K}$ with a single transient per data point. The build-up of ${ }^{13} \mathrm{C}$ polarization was measured by using: Black curve: A stateof-the-art and high-power CP $r f$-pulse sequence described in the Supporting Information (SI); and Grey curve: The low-power multi- $d \mathrm{CP} r f$-pulse sequence described in Figure 1. The traces have the same overall form, and plateau over a period of $\sim 1500 \mathrm{~s}$. The $d \mathrm{CP}$ and $\mathrm{CP}$ build-up curves were fitted with a monoexponential build-up function $\mathrm{A}(1-\exp \{-\mathrm{t} / \tau\})$ using the build-up time constants $\tau=\tau_{\mathrm{CP}}$ and $\tau=\tau_{d \mathrm{CP}}$, respectively. Build-up time constants: Black dashed curve $(\mathrm{CP}): \tau_{\mathrm{CP}}=4.2 \pm 0.2$ minutes; Grey dashed curve $(d \mathrm{CP}): \tau_{d \mathrm{CP}}=5.1 \pm 0.2$ minutes.

The ${ }^{13} \mathrm{C}$ nuclear spin polarization level achieved by the multi- $d \mathrm{CP} r f$-pulse sequence is $\sim 32.2 \%$ after 7 minutes (2 transfer steps) and ultimately reaches $\sim 43.5 \%$ with a build-up time constant $\tau_{d \mathrm{CP}}=5.1 \pm 0.2$ minutes, see Figure 2 (grey curve). The very first ${ }^{1} \mathrm{H} \rightarrow{ }^{13} \mathrm{C}$ nuclear polarization transfer step alone achieves a ${ }^{13} \mathrm{C}$ polarization of $\sim 21.2 \%$ after only 3.5 minutes. A multiple-step CP rf-pulse sequence (see the Supporting Information (SI) for more details) obtains a maximum ${ }^{13} \mathrm{C}$ polarization level of $\sim 63.3 \%$ under the same experimental conditions (black curve). The overall performance of the multi$d \mathrm{CP} r f$-pulse sequence compared with a sophisticated and highpower multiple-contact CP rf-pulse sequence is $\sim 0.69$, which is determined from integrals of the multiple-contact $\mathrm{CP}$ and multi$d \mathrm{CP}{ }^{13} \mathrm{C}$ NMR signal maxima. The grey curve in Figure 2 also 
shows a small decrease in ${ }^{13} \mathrm{C}$ polarization following each transfer step. ${ }^{13} \mathrm{C}$ NMR spectra acquired around these regions are shown in the SI.

Polarized samples of I were dissolved with $5 \mathrm{~mL}$ of $\mathrm{D}_{2} \mathrm{O}$ solvent prepressurized at 6 bar, and subsequently heated to $180^{\circ} \mathrm{C}$ (and a pressure of 9 bar). The liquid sample was transferred in $10 \mathrm{~s}$ to a Bruker Biospin prototype $d \mathrm{DNP}$ injector placed in the bore of a $1.88 \mathrm{~T}\left({ }^{1} \mathrm{H}\right.$ nuclear Larmor frequency $=$ $80.05 \mathrm{MHz},{ }^{13} \mathrm{C}$ nuclear Larmor frequency $=20.13 \mathrm{MHz}$ ) permanent magnet Bruker Biospin Fourier 80 benchtop NMR system by pushing with helium gas at 6 bar through a PTFE tube (1.6 mm inner diameter) running inside a series of solenoid coils ( 2 A power source) producing a minimum magnetic field of 4 $\mathrm{mT}$ along the entire sample transfer path ( $2.8 \mathrm{~m}$ length), see Figure $3 \mathrm{a}$ [37]. Experimental liquid-state ${ }^{1} \mathrm{H}$ and ${ }^{13} \mathrm{C} \mathrm{NMR}$ data were recorded and processed using TopSpin 4.0 software.

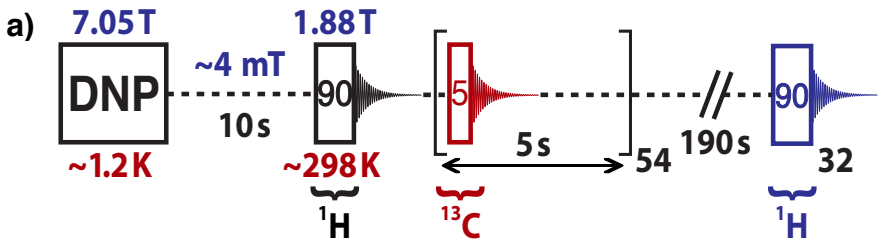

b)

\section{H Hyperpolarized}

${ }^{1} \mathrm{H}$ Thermal Equilibrium $(\times 512)$

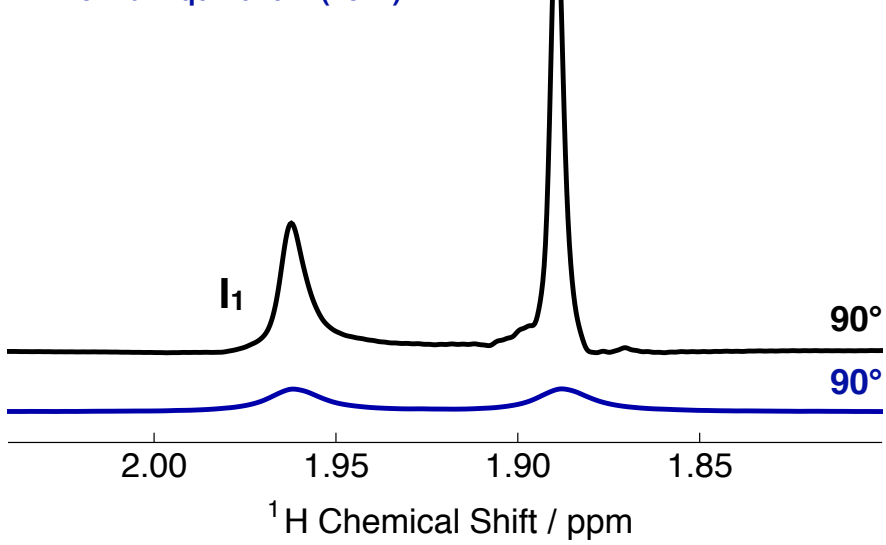

c)

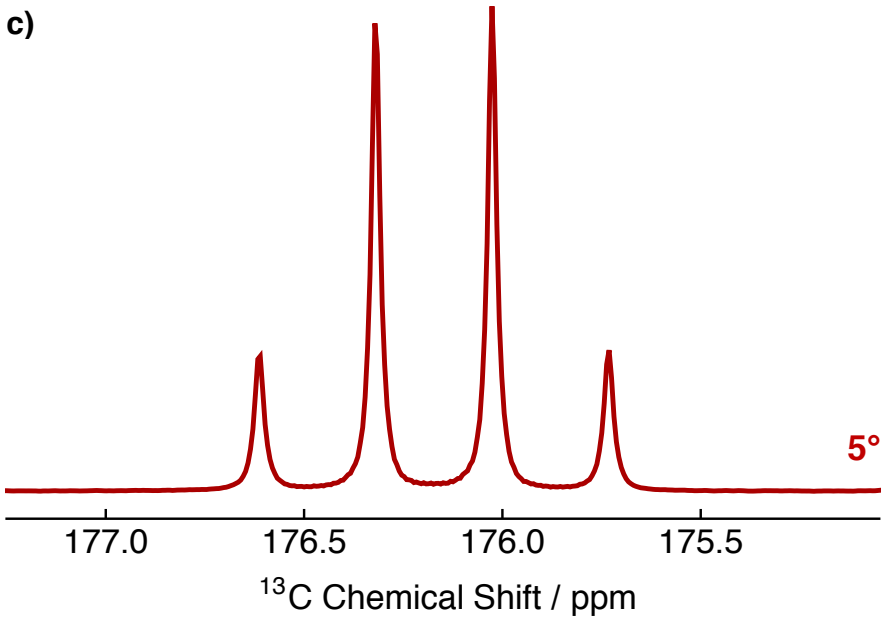

Figure 3: (a) Events and timings for DNP, sample dissolution and acquisition of liquid-state NMR spectra. Relevant portions of the experimental (b) ${ }^{1} \mathrm{H}$ and (c) ${ }^{13} \mathrm{C}$ NMR spectra belonging to the methyl $\left(\mathrm{CH}_{3}\right)$ group and ${ }^{13} \mathrm{C}$-labelled carbonyl site, respectively, of $\mathbf{I}$ in approximately $0.6 \mathrm{~mL}$ of $\mathrm{D}_{2} \mathrm{O}$ solution acquired at $1.88 \mathrm{~T}\left({ }^{1} \mathrm{H}\right.$ nuclear Larmor frequency $=80.05 \mathrm{MHz},{ }^{13} \mathrm{C}$ nuclear Larmor frequency $=20.13 \mathrm{MHz}$ ) and $298.15 \mathrm{~K}$. The experimental NMR spectra were acquired in accordance with the events and timings depicted in (a). $\mathrm{I}_{1}$ and $\mathrm{I}_{2}$ refer to the ${ }^{1} \mathrm{H}$ NMR signal integrals of the multiplet lineshape components.
The first experimental ${ }^{13} \mathrm{C}$ NMR spectrum of the small flip-angle $r f$-pulse and acquire train in (a) is shown in (c).

The sequence of events given in Figure 3a details how the experimental NMR spectra were acquired. After DNP and sample dissolution and transfer, a $90^{\circ} \mathrm{rf}$-pulse is used to record a hyperpolarized ${ }^{1} \mathrm{H}$ NMR spectrum (black). Subsequently, a train of $5^{\circ} r f$-pulses separated by $5 \mathrm{~s}$ is used to record the hyperpolarized ${ }^{13} \mathrm{C}$ NMR spectra (red). The sample is allowed to rest in the $1.88 \mathrm{~T}$ magnet for an additional $190 \mathrm{~s}$ in order to achieve thermal equilibrium, and a proton NMR signal is acquired using a $90^{\circ} \mathrm{rf}$-pulse (blue).

The black spectrum in Figure $3 \mathrm{~b}$ shows the relevant region of the experimental ${ }^{1} \mathrm{H}$ NMR spectrum of $\mathbf{I}$ acquired immediately after sample dissolution and transfer. The sample was initially hyperpolarized in the solid-state by using the multi$d \mathrm{CP} r f$-pulse sequence. The methyl $\left(\mathrm{CH}_{3}\right)$ group resonance located at $\sim 1.93 \mathrm{ppm}$ is split into two peaks due to a scalar coupling with the ${ }^{13} \mathrm{C}$-labelled carbonyl site (scalar coupling constant: $\mid{ }^{2} J_{\mathrm{HC}} \simeq 5.8 \mathrm{~Hz}$ ).

The proton NMR lineshape in Figure $3 \mathrm{~b}$ is highly asymmetric, and indicates that the scalar coupled ${ }^{13} \mathrm{C}$ nuclear spins within the sample are significantly hyperpolarized. The degree of multiplet asymmetry can be used to infer the ${ }^{13} \mathrm{C}$ polarization of the sample upon arrival inside the receiving magnet, a methodology known as SPY-MR [38]. The ${ }^{13} \mathrm{C}$ polarization level $P\left({ }^{13} \mathrm{C}\right)$ is given by the following expression:

$P\left({ }^{13} \mathrm{C}\right)=\frac{\mathrm{I}_{1}-\mathrm{I}_{2}}{\mathrm{I}_{1}+\mathrm{I}_{2}} \times 100 \%(1)$

where $I_{1}$ and $I_{2}$ are the ${ }^{13} \mathrm{C}$ NMR signal integrals of the most and least intense multiplet peaks, respectively. In the case of the ${ }^{1} \mathrm{H}$ NMR spectrum presented in Figure 3b, by using Equation 1 it can be deduced that the ${ }^{13} \mathrm{C}$ polarization $P\left({ }^{13} \mathrm{C}\right)$ is $\sim 28.5 \%$. The hyperpolarized ${ }^{13} \mathrm{C}$ NMR spectrum (Figure $3 \mathrm{c}$ ) was also compared indirectly to the thermal equilibrium ${ }^{1} \mathrm{H}$ NMR spectrum of I (Figure 3b, blue spectrum), with a $0.2 \%$ agreement on the final ${ }^{13} \mathrm{C}$ polarization.

The ${ }^{13} \mathrm{C}$ NMR spectrum acquired immediately after sample dissolution and transfer is shown in Figure $3 \mathrm{c}$. The ${ }^{13} \mathrm{C}$ carbonyl resonance of $I$ is positioned at $\sim 176.2 \mathrm{ppm}$ and displays a 1:3:3:1 quartet structure due to the $\left.\right|^{2} J_{\mathrm{HC}} \mid$ scalar coupling with the 3 methyl group protons. The signal-to-noise ratio (SNR) of the $\mathrm{NMR}$ peak belonging to the ${ }^{13} \mathrm{C}$-labelled site was determined to be $\sim 1905$.

The longitudinal relaxation time constant $T_{1}$ of the ${ }^{13} \mathrm{C}$ labelled carbonyl site in $\mathbf{I}$ was measured by applying a small flip-angle $r f$-pulse $\left(5^{\circ}\right)$ followed directly by ${ }^{13} \mathrm{C}$ NMR signal detection (acquisition time $=3 \mathrm{~s}$ ) every $5 \mathrm{~s}$, see Figure $3 \mathrm{a}$. The resulting curve was found to have a single exponential relaxation behaviour (data not shown), and is well fitted with a mono-exponential decay function using a sole relaxation time constant $T_{1}$. Mono-exponential decay function: $\operatorname{Aexp}\left\{-\mathrm{t} / T_{1}\right\}$. The value of $T_{1}$ was determined to be: $99.0 \pm 0.7 \mathrm{~s}$, taking into account the influence of the small flip-angle $r f$-pulse train.

The relevant portion of the experimental ${ }^{1} \mathrm{H}$ NMR spectrum belonging to the methyl $\left(\mathrm{CH}_{3}\right)$ group protons of $\mathbf{I}$ acquired under thermal equilibrium conditions is also shown in Figure $3 \mathrm{~b}$ (blue spectrum). This spectrum allows an estimate of the resulting liquid-state sample concentration for $\mathbf{I}$ after dissolution and transfer by comparing the ${ }^{1} \mathrm{H}$ NMR signal integral to that from a sample of $I$ at a known concentration. The 
solution-state sample concentration of I was consequently found to be: $\sim 14.7 \mathrm{mM}$.

In the solid-state, the multiple-contact $\mathrm{CP} r f$-pulse sequence is clearly superior in terms of ${ }^{1} \mathrm{H} \rightarrow{ }^{13} \mathrm{C}$ polarization transfer and achieves a higher final ${ }^{13} \mathrm{C}$ polarization before sample dissolution. A suspected reason for the lower efficiency of $d \mathrm{CP}$ polarization transfer is the quantity of ${ }^{1} \mathrm{H}$ polarization depleted to complete the $d \mathrm{CP}$ transfer step compared with the $\mathrm{CP} r f$-pulse sequence. Proton NMR spectra showing this effect are given in the SI. It was found that a CP contact retains $\sim 82.9 \%$ of the initial ${ }^{1} \mathrm{H}$ polarization, whereas the $d \mathrm{CP} r f$-pulse sequence retains only $\sim 19.8 \%$. Consequently, there is less ${ }^{1} \mathrm{H}$ polarization available at subsequent polarization transfer steps. This result very likely inhibits the efficiency of the remaining polarization transfer stages in the $d \mathrm{CP} r f$-pulse sequence. This effect should be investigated in the future, and the inter- $d \mathrm{CP}$ delays further optimized (and likely lengthened) to ultimately increase the overall $d \mathrm{CP}$ efficiency, since the current inter- $d \mathrm{CP}$ delays were intentionally matched to those of a fully optimized $\mathrm{CP}$ experiment. Liquid-state ${ }^{13} \mathrm{C}$ polarizations on the order of $\sim 40 \%$ have previously been demonstrated for $\left[1-{ }^{13} \mathrm{C}\right]$ sodium acetate by using a multiple-contact $\mathrm{CP} r f$-pulse sequence prior to sample dissolution $[39,40]$. Nevertheless, a liquid-state ${ }^{13} \mathrm{C}$ polarization of $\sim 28.5 \%$ is encouraging given the initial solidstate ${ }^{13} \mathrm{C}$ polarization of $\sim 43.5 \%$.

SPY-MR polarimetry [38] in liquid-state NMR works in the case that the nuclear spins involved are not participating in strong coupling (which cannot be the case for heteronuclear spins at sufficiently high magnetic fields). As a result; the SPYMR approach was implemented to infer the level of ${ }^{13} \mathrm{C}$ polarization from the hyperpolarized ${ }^{1} \mathrm{H}$ NMR spectrum in the liquid-state after dissolution. This is a feasible approach since the lower sensitivity of our benchtop magnet, with respect to higher magnetic field superconducting instruments, requires very long (on the order of days) accumulations of ${ }^{13} \mathrm{C}$ thermal equilibrium spectra in order to obtain an NMR signal with a sufficient signal-to-noise ratio (SNR).

There is evidentially a discrepancy between solid-state and liquid-state ${ }^{13} \mathrm{C}$ polarizations. Given the long $T_{1}$ of sample $\mathbf{I}$ at low magnetic field, it is unlikely that solely ${ }^{13} \mathrm{C}$ nuclear spin relaxation is responsible for the difference in results. Another possibility is that ${ }^{1} \mathrm{H}-{ }^{13} \mathrm{C}$ dipolar order is generated and survives the dissolution process and is not eradicated by changing magnetic field gradients during the sample transfer step to the detection magnet. The presence of such dipolar order would limit the applicability of the SPY-MR approach [38]. The excessive losses in ${ }^{13} \mathrm{C}$ polarization are not accounted for at present and are largely thought to be related to the sample dissolution and transfer processes but also could be attributable to zero or double quantum coherences created in regions of low magnetic field and incoherent cross-relaxation phenomena [41].

An $r f$-pulse sequence which employs low-power $r f$-pulses for the stepwise transfer of ${ }^{1} \mathrm{H}$ polarization to ${ }^{13} \mathrm{C}$ nuclear spins under $d \mathrm{DNP}$ conditions was demonstrated. The multi- $d \mathrm{CP} r f$ sequence achieves a ${ }^{13} \mathrm{C}$ polarization level of $\sim 43.5 \%$ in the solid-state after $\sim 25$ minutes ( 7 polarization transfer steps). The overall $d \mathrm{CP}$ polarization transfer efficiency of the approach was found to be $\sim 0.69$ with respect to a sophisticated and highpower multiple-contact CP experiment. After dissolution with a hot solvent, hyperpolarized liquid-state NMR signals were detected and a ${ }^{13} \mathrm{C}$ polarization of $\sim 28.5 \%$ was observed in a separate permanent magnet benchtop NMR system. These results are promising for future applications of indirect hyperpolarization techniques and dissolution of insensitive nuclear spins. The low-power nature of the multi-dCP approach may allow polarization transfer techniques to be implemented in larger sample volumes, paving the way to the use of indirect ${ }^{1} \mathrm{H} \rightarrow{ }^{13} \mathrm{C}$ polarization transfer schemes in (pre)clinical settings.

\section{Experimental Methods}

Sample Preparation. A solution of $3 \mathrm{M}\left[1-{ }^{13} \mathrm{C}\right]$ sodium acetate in the glass-forming mixture $\mathrm{H}_{2} \mathrm{O}: \mathrm{D}_{2} \mathrm{O}$ :glycerol- $d_{8}$ (10\%:30\%:60\% v/v/v) was doped with $30 \mathrm{mM}$ TEMPOL radical (all compounds purchased from Sigma Aldrich) and sonicated for $\sim 10$ minutes. This sample is referred to as $\mathbf{I}$ in the main text. Paramagnetic TEMPOL radicals were chosen to most efficiently polarize ${ }^{1} \mathrm{H}$ spins under our $d \mathrm{DNP}$ conditions.

Sample Freezing. $100 \mu \mathrm{L}$ sample volumes were pipetted into a PEEK sample cup and inserted into a $7.05 \mathrm{~T}$ prototype Bruker Biospin polarizer equipped with a specialized $d \mathrm{DNP}$ probe and running TopSpin 3.5 software. The sample temperature was reduced to $1.2 \mathrm{~K}$ by submerging the sample in liquid helium and reducing the pressure of the variable temperature insert (VTI) towards $\sim 0.7$ mbar.

\section{Supporting Information}

The Supporting Information is available free of charge. Multiple-contact CP $r f$-pulse sequence in Figure $\mathrm{S} 1,{ }^{1} \mathrm{H}$ detected NMR spectra after $d \mathrm{CP}$ and $\mathrm{CP}$ polarization transfer steps in Figure $\mathrm{S} 2$, and ${ }^{13} \mathrm{C}$ detected $\mathrm{NMR}$ spectra around $d \mathrm{CP}$ polarization transfer steps in Figure S3.

\section{Conflict of Interest}

The authors declare no conflict of interest.

\section{Acknowledgements}

This research was supported by ENS-Lyon, the French CNRS, Lyon 1 University, the European Research Council under the European Union's Horizon 2020 research and innovation program (ERC Grant Agreements No. 714519 / HP4all and Marie Skłodowska-Curie Grant Agreement No. 766402 / ZULF). The authors gratefully acknowledge Bruker Biospin for providing the prototype $d \mathrm{DNP}$ polarizer and injector, and particularly Dmitry Eshchenko, Roberto Melzi, Marc Rossire, Marco Sacher, Marc Schnell and James Kempf for scientific and technical support. The authors graciously acknowledge Frank Decker and Bruno Knittel for lending assistance with the operation of the permanent magnet Bruker Biospin Fourier 80 benchtop NMR system. The authors additionally acknowledge Catherine Jose and Christophe Pages for use of the ISA Prototype Service; Stéphane Martinez of the UCBL mechanical workshop for machining parts of the experimental apparatus; Paul-Emmanuel Edeline for assisting with dissolution experiments; Burkhard Luy, Malcolm H. Levitt and Christian Bengs for fruitful discussions; and Gerd Buntkowsky who kindly communicated data associated with prior publications to us.

\section{References}

[1] J.-H. Ardenkjær-Larsen, B. Fridlund, A. Gram, G. Hansson, L. Hansson, M. H. Lerche, R. Servin, M. Thaning and K. Golman, Increase in signal-to-noise ratio of $>10,000$ times in liquid-state NMR, Proc. Natl. Acad. Sci. U.S.A., 2003, 100, 10158-10163.

[2] S. E. Day, M. I. Kettunen, F. A. Gallagher, D.-E. Hu, M. Lerche, J. Wolber, K. Golman, J. H. Ardenkjær-Larsen and K. M. Brindle, Detecting tumor response to treatment using hyperpolarized ${ }^{13} \mathrm{C}$ magnetic resonance imaging and spectroscopy, Nat. Med., 2007, 13, 1382-1387.

[3] K. M. Brindle, S. E. Bohndiek, F. A. Gallagher and M. I. Kettunen, Tumor imaging using hyperpolarized ${ }^{13} \mathrm{C}$ magnetic resonance spectroscopy, Magn. Reson. Med., 2011, 66, 505-519. 
[4] S. J. Nelson, J. Kurhanewicz, D. B. Vigneron, P. E. Z. Larson, A. L. Harzstark, M. Ferrone, M. van Criekinge, J. W. Chang, R. Bok, I. Park, G. Reed, L. Carvajal, E. J. Small, P. Munster, V. K. Weinberg, J. H. Ardenkjær-Larsen, A. P. Chen, R. E. Hurd, L.-I. Odegardstuen, F. J. Robb, J. Tropp and J. A. Murray, Metabolic imaging of patients with prostate cancer using hyperpolarized $\left[1{ }^{13} \mathrm{C}\right]$ pyruvate, Sci. Transl. Med., 2013, 5, 198.

[5] A. Bornet, X. Ji, D. Mammoli, B. Vuichoud, J. Milani, G. Bodenhausen and S. Jannin, Long-Lived States of Magnetically Equivalent Spins Populated by Dissolution-DNP and Revealed by Enzymatic Reactions, Chem.: Eur. J., 2014 20, 17113-17118.

[6] J.-N. Dumez, J. Milani, B. Vuichoud, A. Bornet, J. Lalande-Martin, I. Tea, M. Yon, M. Maucourt, C. Deborde, A. Moing, L. Frydman, G. Bodenhausen, S. Jannin and P. Giraudeau, Hyperpolarized NMR of plant and cancer cell extracts at natural abundance, Analyst, 2015, 140, 5860-5863.

[7] A. Bornet, M. Maucourt, C. Deborde, D. Jacob, J. Milani, B. Vuichoud, X. Ji, J.-N. Dumez, A. Moing, G. Bodenhausen, S. Jannin and P. Giraudeau, Highly Repeatable Dissolution Dynamic Nuclear Polarization for Heteronuclear NMR Metabolomics, Anal. Chem., 2016, 88, 6179-6183.

[8] S. Jannin, J.-N. Dumez, P. Giraudeau and D. Kurzbach, Application and methodology of dissolution dynamic nuclear polarization in physical, chemical and biological contexts, J. Magn. Reson., 2019, 305, 41-50.

[9] S. Jannin, A. Bornet, S. Colombo and G. Bodenhausen, Low-temperature cross polarization in view of enhancing dissolution Dynamic Nuclear Polarization in NMR, Chem. Phys. Lett., 2011, 517, 234-236.

[10] S. R. Hartmann and E. L. Hahn, Nuclear Double Resonance in the Rotating Frame, Phys. Rev., 1962, 128, 204-205.

[11] A. Pines, M. Gibby and J. Waugh, Proton-enhanced nuclear induction spectroscopy ${ }^{13} \mathrm{C}$ chemical shielding anisotropy in some organic solids, Chem Phys. Lett., 1972, 15, 373-376.

[12] A. Bornet, R. Melzi, S. Jannin and G. Bodenhausen, Cross Polarization for Dissolution Dynamic Nuclear Polarization Experiments at Readily Accessible Temperatures $1.2<\mathrm{T}<4.2 \mathrm{~K}$, Appl. Magn. Reson., 2012, 43, 107-117.

[13] M. Batel, M. Krajewski, A. Däpp, A. Hunkeler, B. H. Meier, S. Kozerke and M. Ernst, Cross Polarization for Dissolution Dynamic Nuclear Polarization, Chem. Phys. Lett., 2012, 554, 72-76.

[14] A. Bornet, R. Melzi, A. J. Perez Linde, P. Hautle, B. van den Brandt, S. Jannin and G. Bodenhausen, Boosting Dissolution Dynamic Nuclear Polarization by Cross Polarization, J. Chem. Phys. Lett., 2013, 4, 111-114.

[15] B. Vuichoud, A. Bornet, F. de Nanteuil, J. Milani, E. Canet, X. Ji, P. Miéville, E. Weber, D. Kurzbach, A. Flamm, R. Konrat, A. D. Gossert, S. Jannin and G. Bodenhausen, Filterable Agents for Hyperpolarization of Water, Metabolites and Proteins, Chem.: Eur. J., 2016, 22, 14696-14700.

[16] M. Cavaillès, A. Bornet, X. Jaurand, B. Vuichoud, D. Baudouin, M. Baudin, L. Veyre, G. Bodenhausen, J.-N. Dumez, S. Jannin, C. Copéret and C. Thieuleux, Tailored Microstructured Hyperpolarizing Matrices for Optima Magnetic Resonance Imaging, Angew. Chem. Int. Ed., 2018, 130, 7575-7579.

[17] A. J. Perez Linde, PhD thesis, University of Nottingham, UK, 2009.

[18] G. Hartmann, D. Hubert, S. Mango, C. C. Morehouse and K. Plog, Proton polarization in alcohols at $50 \mathrm{kG}, 1 \mathrm{~K}$, Nucl. Instrum. Meth. A, 1973, 106, 9-12. [19] X. Ji, A. Bornet, B. Vuichoud, J. Milani, D. Gajan, A. J. Rossini, L. Emsley, G. Bodenhausen and S. Jannin, Transportable Hyperpolarized Metabolites, Nat. Commun., 2017, 8, 13975 .

[20] J. M. O. Vinther, V. Zhurbenko, M. M. Albannay and J.-H. ArdenkjærLarsen, Design of a local quasi-distributed tuning and matching circuit for dissolution DNP cross polarization, Solid State Nucl. Mag., 2019, 102, 12-20.

[21] K. W. Lipsø, S. Bowen, O. Rybalko and J.-H. Ardenkjær-Larsen, Large dose hyperpolarized water with dissolution-DNP at high magnetic field, $J$. Magn. Reson., 2017, 274, 65-72.

[22] S. J. Elliott, S. F. Cousin, Q. Chappuis, O. Cala, M. Ceillier, A. Bornet and S. Jannin, Dipolar order mediated ${ }^{1} \mathrm{H} \rightarrow{ }^{13} \mathrm{C}$ cross-polarization for dissolutiondynamic nuclear polarization, Magn. Reson., 2020, 1, 89-96.

[23] J. Jeener and P. Broekaert, Nuclear Magnetic Resonance in Solids: Thermodynamic Effects of a Pair of rf Pulses, Phys. Rev., 1967, 157, 232-240. [24] H.-M. Vieth and C. S. Yannoni, Cross polarization in solid state NMR spectroscopy. Efficient polarization transfer via the non-Zeeman spin reservoir, Chem. Phys. Lett., 1993, 205, 153-156.

[25] N. D. Kurur and G. Bodenhausen, Adiabatic Coherence Transfer in Magnetic Resonance of Homonuclear Scalar-Coupled Systems, J. Magn Reson. A, 1995, 114, 163-173.

[26] S. Emid, J. Konijnendijk, J. Smidt and A. Pines, On the short time behavior of the dipolar signal in relaxation measurements by the pulse method, Physica $B+C, \mathbf{1 9 8 0}, 100,215-218$.

[27] S. Zhang, E. Stejskal, R. Fornes and X. Wu, Mismatching Cross Polarization in the Rotating Frame, J. Magn. Reson., Ser A, 1993, 104, 177-179. [28] A. K. Khitrin, J. Xu and A. Ramamoorthy, Cross-correlations between low$\gamma$ nuclei in solids via a common dipolar bath, J. Magn. Reson., 2011, 212, $95-$ 101.

[29] J. Jeener, R. Du Bois and P. Broekaert, "Zeeman" and "Dipolar" Spin Temperatures during a Strong rf Irradiation, Phys. Rev., 1965, 139, A1959A1961.
[30] A. G. Redfield, Nuclear spin thermodynamics in the rotating frame, Science, 1969, 164, 1015-1023.

[31] D. E. Demco, J. Tegenfeldt and J. S. Waugh, Dynamics of cross relaxation in nuclear magnetic double resonance, Phys. Rev. B, 1975, 11, 4133-4151.

[32] M. Kunitomo, H. Hatanaka and T. Hashi, Adiabatic demagnetization in the rotating frame by non-resonant oscillating field, Phys. Lett. A, 1974, 49, $135-$ 136.

[33] J.-S. Lee and A. K. Khitrin, Thermodynamics of adiabatic cross polarization, J. Chem. Phys., 2008, 128, 114504.

[34] A. Bornet, J. Milani, B. Vuichoud, A. J. Perez Linde, G. Bodenhausen and S. Jannin, Microwave frequency modulation to enhance Dissolution Dynamic Nuclear Polarization, Chem. Phys. Lett., 2014, 602, 63-67.

[35] A. Bornet, A. Pinon, A. Jhajharia, M. Baudin, X. Ji, L. Emsley, G. Bodenhausen, J.-H. Ardenkjær-Larsen and S. Jannin, Microwave-gated dynamic nuclear polarization, Phys. Chem. Chem. Phys., 2016, 18, 3053030535 .

[36] S. J. Elliott, O. Cala, Q. Chappuis, S. F. Cousin, D. Eshchenko, R. Melzi, J. G. Kempf and S. Jannin, Submitted, 2021.

[37] J. Milani, B. Vuichoud, A. Bornet, P. Miéville, R. Mottier, S. Jannin and G. Bodenhausen, A magnetic tunnel to shelter hyperpolarized fluids, Rev. Sci. Instrum., 2015, 80, 024101 .

[38] B. Vuichoud, J. Milani, Q. Chappuis, A. Bornet, G. Bodenhausen and S. Jannin, Measuring absolute spin polarization in dissolution-DNP by Spin PolarimetrY Magnetic Resonance (SPY-MR), J. Magn. Reson., 2015, 260, 127135.

[39] B. Vuichoud, J. Milani, A. Bornet, R. Melzi, S. Jannin and G. Bodenhausen, Hyperpolarization of Deuterated Metabolites via Remote CrossPolarization and Dissolution Dynamic Nuclear Polarization, J. Phys. Chem. B 2014, 118, 1411-1415.

[40] A. Bornet and S. Jannin, Optimizing dissolution dynamic nuclear polarization, J. Magn. Reson., 2016, 264, 13-21.

[41] J. E. Ollerenshaw, V. Tugarinov and L. W. Kay, Magn. Reson. Chem., 2003, $41,843-852$. 